\title{
A Pattern-Based Approach Combining Tumor Morphology on MRI With Distinct Signal Patterns on Diffusion-Weighted Imaging to Assess Response of Rectal Tumors After Chemoradiotherapy
}

Citation for published version (APA):

Lambregts, D. M. J., Delli Pizzi, A., Lahaye, M. J., van Griethuysen, J. J. M., Maas, M., Beets, G. L., Bakers, F. C. H., \& Beets-Tan, R. G. H. (2018). A Pattern-Based Approach Combining Tumor Morphology on MRI With Distinct Signal Patterns on Diffusion-Weighted Imaging to Assess Response of Rectal Tumors After Chemoradiotherapy. Diseases of the Colon \& Rectum, 61(3), 328-337. https://doi.org/10.1097/DCR.0000000000000915

Document status and date:

Published: 01/03/2018

DOI:

10.1097/DCR.0000000000000915

Document Version:

Publisher's PDF, also known as Version of record

Document license:

Taverne

Please check the document version of this publication:

- A submitted manuscript is the version of the article upon submission and before peer-review. There can be important differences between the submitted version and the official published version of record. People interested in the research are advised to contact the author for the final version of the publication, or visit the DOI to the publisher's website.

- The final author version and the galley proof are versions of the publication after peer review.

- The final published version features the final layout of the paper including the volume, issue and page numbers.

Link to publication

\footnotetext{
General rights rights.

- You may freely distribute the URL identifying the publication in the public portal. please follow below link for the End User Agreement:

www.umlib.nl/taverne-license

Take down policy

If you believe that this document breaches copyright please contact us at:

repository@maastrichtuniversity.nl

providing details and we will investigate your claim.
}

Copyright and moral rights for the publications made accessible in the public portal are retained by the authors and/or other copyright owners and it is a condition of accessing publications that users recognise and abide by the legal requirements associated with these

- Users may download and print one copy of any publication from the public portal for the purpose of private study or research.

- You may not further distribute the material or use it for any profit-making activity or commercial gain

If the publication is distributed under the terms of Article 25fa of the Dutch Copyright Act, indicated by the "Taverne" license above,

Download date: 26 Apr. 2023 


\title{
A Pattern-Based Approach Combining Tumor Morphology on MRI With Distinct Signal Patterns on Diffusion-Weighted Imaging to Assess Response of Rectal Tumors After Chemoradiotherapy
}

\author{
Doenja M.J. Lambregts, M.D., Ph.D ${ }^{1}$ Andrea Delli Pizzi, M.D. ${ }^{1,2}$ \\ Max J. Lahaye, M.D., Ph.D. ${ }^{1}$ Joost J.M. van Griethuysen, M.D. ${ }^{1,3}$ \\ Monique Maas, M.D., Ph.D. ${ }^{1}$ - Geerard L. Beets, M.D., Ph.D.,4 \\ Frans C.H. Bakers, M.D. ${ }^{5}$ Regina G.H. Beets-Tan, M.D., Ph.D ${ }^{1,3}$ \\ 1 Department of Radiology, Netherlands Cancer Institute, Amsterdam, The Netherlands \\ 2 Department of Neuroscience and Imaging, Gabriele d'Annunzio University, SS Annunziate Hospital, Chieti, Italy \\ 3 GROW School for Oncology and Developmental Biology, Maastricht University, Maastricht, The Netherlands \\ 4 Department of Surgery, Netherlands Cancer Institute, Amsterdam, The Netherlands \\ 5 Department of Radiology, Maastricht University Medical Centre, Maastricht, The Netherlands
}

BACKGROUND: Diffusion-weighted imaging is increasingly used in rectal cancer MRI to assess response after chemoradiotherapy. Certain pitfalls (eg, artefacts) may hamper diffusion-MRI assessment, leading to suboptimal diagnostic performance. Combining diffusion-weighted MRI with the underlying morphology on standard (T2-weighted) MRI may help overcome these pitfalls.

OBJECTIVE: The purpose of this study was to evaluate the diagnostic performance of a pattern-based approach combining tumor morphology on T2-weighted MRI with distinct diffusion-weighted imaging signal patterns to assess response after chemoradiotherapy in rectal cancer.

DESIGN: Response to chemoradiotherapy was scored according to 4 patterns: 1) cases with either a clear residual mass with corresponding high-diffusion signal $(\mathrm{A}+)$ or completely normalized wall without diffusion signal (A-);2) cases with circular and/or irregular

Funding/Support: None reported.

Financial Disclosure: None reported.

Correspondence: Doenja M.IJ. Lambregts, M.D., Ph.D., Department of Radiology, Netherlands Cancer Institute, PO Box 90203, 1006 BE Amsterdam, The Netherlands. E-mail: d.lambregts@nki.nl or doenja. lambregts@gmail.com

Dis Colon Rectum 2018; 61: 328-337

DOI: $10.1097 /$ DCR.0000000000000915

(c) The ASCRS 2018 fibrosis with (B+) or without (B-) small foci of diffusion signal scattered throughout the fibrosis; 3 ) cases with semicircular fibrosis with (C+) or without (C-) high diffusion signal at the inner margin of the fibrosis; and 4) polypoid tumors showing regression of the polyp and fibrosis at the site of the stalk with (D+) or without (D-) focal high-diffusion signal in the stalk. A total of 75 cases were rescored by an independent second reader to study interobserver variations. Standard of reference was histopathology or long-term outcome.

SETTINGS: The study was conducted at a single tertiary referral center.

PATIENTS: A total of 222 patients with locally advanced rectal cancer undergoing chemoradiotherapy were included.

MAIN OUTCOME MEASURES: Diagnostic performance to discriminate between a complete response and residual tumor was measured.

RESULTS: The pattern-based approach resulted in a sensitivity of $94 \%$, specificity of $77 \%$, positive predictive value of $88 \%$, negative predictive value of $87 \%$, and overall accuracy of $88 \%$ to differentiate between tumor versus complete response. Accuracies per pattern were $100 \%$ (A), 74\% (B), 86\% (C), and 92\% (D). Interobserver agreement was good $(\kappa=0.75)$.

LIMITATIONS: The study included no comparison with routine (nonpattern) diffusion-MRI assessment.

CONCLUSIONS: A pattern-based approach combining tumor morphology with distinct diffusion-weighted 
imaging patterns results in good diagnostic performance to assess response. See Video Abstract at http://links.lww. com/DCR/A433.

KEY WORDS: Chemoradiotherapy; Diffusion-weighted imaging; Magnetic resonance imaging; Rectal cancer; Response.

I $\mathrm{n}$ recent years, diffusion-weighted imaging (DWI) has increasingly been acknowledged as a beneficial adjunct to the routine MRI protocol for assessing rectal cancer, particularly in the restaging setting after neoadjuvant treatment. DWI uses the differences in the extracellular movement diffusion of water protons to discriminate between tissues of varying cellularity and is known to be a very sensitive technique to detect malignant (hypercellular) tumors. Various studies, including a meta-analysis by van der Paardt et al, ${ }^{1}$ have shown that the addition of DWI can significantly improve the diagnostic performance of MRI for re-evaluation of the local tumor stage after chemoradiotherapy (CRT). ${ }^{2,3}$ In particular, DWI can help discriminate patients with residual viable tumor from patients with a complete tumor response. This is an increasingly relevant clinical issue, given the recent introduction of watchful waiting as a potential alternative to surgical resection for complete responders. ${ }^{4,5}$ To safely select patients for watchful waiting, accurate selection of the true complete responders is crucial.

The generally used principle of assessing DWI is relatively straightforward: the absence of high signal on high b-value DWI is suggestive of a complete tumor regression, whereas a remaining high DWI signal indicates that a tumor remnant is still present. However, there are some potential drawbacks to this approach. A high DWI signal may also be caused by shine-through effects of a high signal in fluids (eg, in the rectal lumen) and by the fact that other anatomic structures with a dense cellular structure (eg, lymph nodes) and inflammatory processes (eg, abscesses, fistulas) may also exhibit a high signal. ${ }^{6}$ Moreover, artefacts caused by the presence of air in the rectal lumen may result in a false high signal projecting over the rectal wall. ${ }^{7}$ To help overcome these pitfalls, it may be helpful to advocate for a more structured, pattern-based approach combining diffusion-weighted signal patterns with the underlying morphology on standard MRI. Such a patternbased reading approach has previously also been shown beneficial for the staging and follow-up of rectal tumors on T2-weighted $\mathrm{MRI}^{8,9}$ and for the assessment of rectal cancer lymph nodes on DWI ${ }^{10}$ but has not yet been investigated for tumor response evaluation on DWI.

The aim of this study was to explore the value of a pattern-based approach to assess rectal tumor response after neoadjuvant treatment (specifically to differentiate between complete responders and patients with residual tumor) using a combination of tumor morphology on standard T2-weighted imaging with distinct signal patterns on DWI.

\section{PATIENTS AND METHODS}

A retrospective analysis of imaging data acquired as part of routine clinical diagnostic imaging procedures was performed. The study was approved by the local institutional review board, and informed consent was waived.

\section{Patients}

A total of 297 consecutive patients with locally advanced rectal cancer treated with a long course of neoadjuvant treatment at Maastricht University Medical Centre between 2008 and 2015 were considered for inclusion. Inclusion criteria consisted of histopathologically proven nonmucinous rectal adenocarcinoma, long-course neoadjuvant treatment, availability of a primary staging MRI and a restaging MRI including a DWI sequence, and data on the final response based on histopathology after surgery or long-term follow-up (in case of a clinical complete response managed with watchful waiting). Seventy-five patients were excluded (see Figure 1). Mucinous tumors were excluded because of their mucin content (causing high-signal T2 shinethrough with different signal characteristics on DI). ${ }^{11}$ Patients with severe artefacts on DWI (eg, caused by metal hip prostheses) were also excluded, because in these cases the rectal wall and thus the response pattern cannot be assessed on DWI. A final total study population of 222 patients remained ( 154 men; median age $=66 \mathrm{y})$. The routine neoadjuvant treatment consisted of 50.4 -Gy radiation combined with $2 \times 825 \mathrm{mg} / \mathrm{m}^{2}$ per day of capecitabine. Twenty-three patients underwent an alternative neoadjuvant treatment scheme (25-Gy radiation followed by a long interval, with or without additional chemotherapy or sirolimus/rapamycin (Rapamune, Wyeth Pharmaceuticals (subsidiary of Pfizer Inc., Philadelphia, USA).

\section{Imaging}

All of the restaging MRIs were performed at $1.5 \mathrm{~T}$ (Intera (Achieva) or Ingenia MR system, Philips Medical Systems, Best, The Netherlands) using a phased array surface coil, routinely performed 6 to 10 weeks after completion of neoadjuvant treatment. To reduce bowel motility, patients received $20 \mathrm{mg}$ of scopolamine butylbromide (Buscopan, Boehringer Ingelheim, Ingelheim am Rhein, Germany) intravenously, either in case of anticipated bowel movement artefacts on the sagittal planning scan (first part of the study period) or routinely (final part of the study period). From March 2014, patients also routinely received a microenema (Microlax, Mc- 


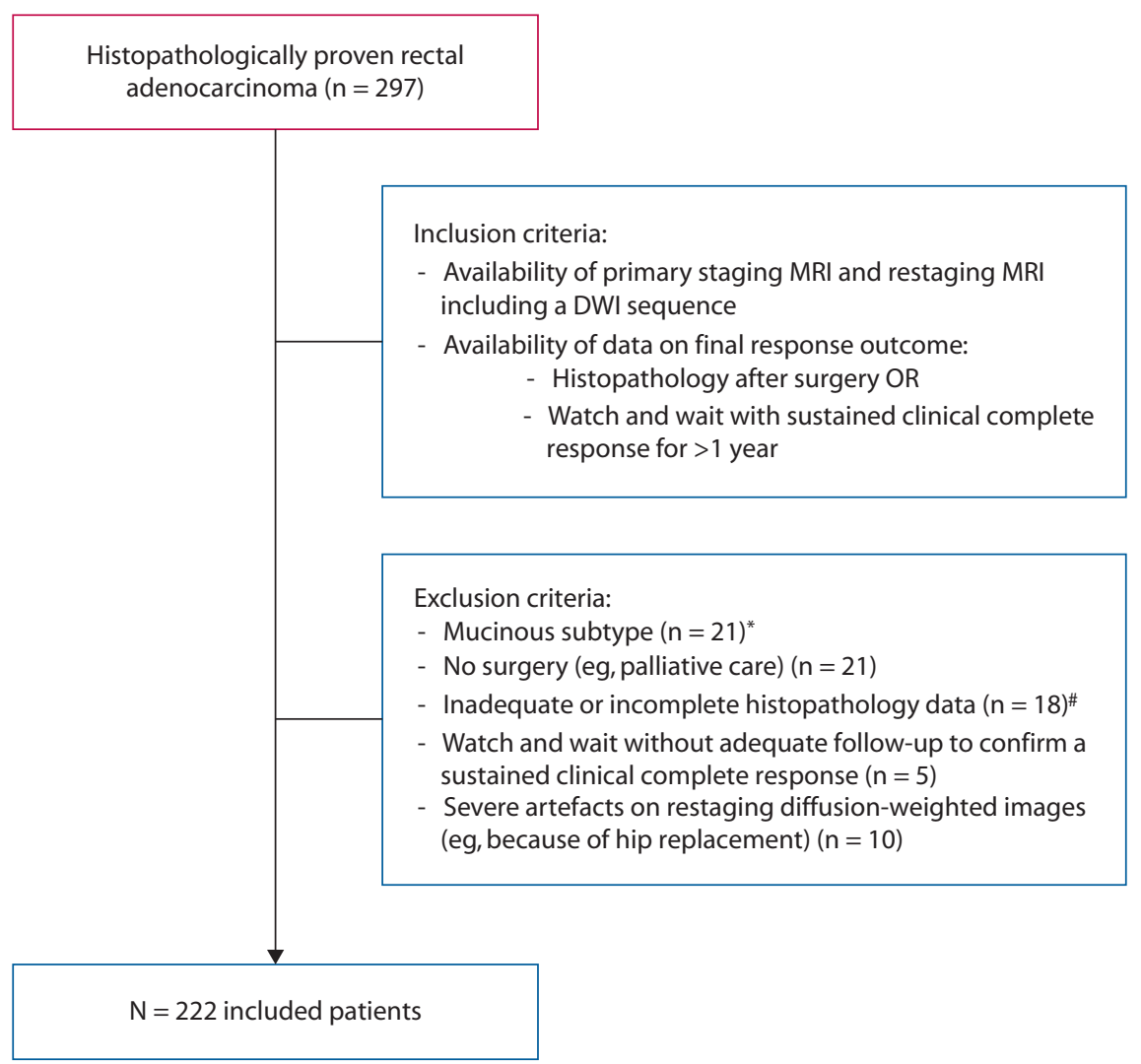

FIGURE 1. Flow chart of patients considered for inclusion, excluded patients, and patients finally included in the study cohort. *Mucinous tumors were excluded, because these are known to exhibit different characteristics on diffusion-weighted MRI. ${ }^{11}$ \#Patients with an interval between restaging MRI and surgery of $>50$ days were excluded. DWI = diffusion-weighted imaging.

Neil Healthcare Limited, Dublin, Ireland) \pm 15 minutes before onset of the examination, to reduce air artefacts. The standard clinical MRI protocol included 2D T2-weighted fast spin echo sequences in 3 planes (sagittal, transverse, and coronal) and a transverse diffusion-weighted sequence (highest b-value b1000): the transverse and coronal planes were angled perpendicular and parallel to the tumor axis as identified on the sagittal planning scan. Apparent diffusion coefficient (ADC) maps were automatically generated at the operating system. Detailed sequence parameters are given in Table 1.

\section{TABLE 1. Sequence parameters of the T2-weighted and DWI sequences used during the study}

\begin{tabular}{|c|c|c|c|c|}
\hline & \multirow[b]{2}{*}{ T2-weighted FSE } & \multicolumn{3}{|c|}{ Diffusion-weighted MRI } \\
\hline & & DWI-1 & DWI-2 & $D W I-3^{a}$ \\
\hline Repetition time & $8456-9558$ & $4808-4829$ & 4971 & $4172-5241$ \\
\hline No. of slices & $22-30$ & 50 & 24 & $20-24$ \\
\hline Slice thickness, mm & $3-5$ & 5 & 5 & 5 \\
\hline FOV, $\mathrm{mm}$ & 200 & 440 & 320 & 320 \\
\hline Echotrain length & 25 & 1 & 1 & 1 \\
\hline No. of signal averages & $2-6$ & 4 & 5 & $4-6$ \\
\hline Acquisition time, min:s & $4: 37-6: 30$ & $10: 37-12: 20$ & 05:33 & 05:51-06:44 \\
\hline B-values & - & $0,(100), 500,1000$ & $0,500,1000$ & $0,(25,50,100), 500,1000,(2000)$ \\
\hline Fat suppression technique & - & STIR & SPIR & SPAIR \\
\hline Echo planar imaging factor & - & $53-55$ & 55 & 61 \\
\hline
\end{tabular}

The DWI-1 sequence was applied from September 2008 to December 2011, the DWI-2 from December 2011 to June 2012, and the DWI-3 from June 2012 until the end of the study period.

$\mathrm{DWI}=$ diffusion-weighted imaging; FOV = field of view; STIR = short TI inversion recovery; SPIR = spectral presaturation with inversion recovery; SPAIR = spectral attenuated inversion recovery; FSE = fast spin echo.

aIn 9 patients, a slightly adjusted protocol was used with a smaller FOV $(180)$ and a reduced number of b-values $(0,1000)$. 


\section{Image Evaluation: Response Patterns}

An experienced reader (DMJL with 7 y specific rectal MRI experience) evaluated the restaging high b-value (b1000) DWIs together with the restaging T2-weighted images, as well as the primary staging MRI to take into account the location and morphology of the tumor before treatment. The ADC maps were also at the reader's disposal to confirm high signal on DWI to be caused by actual restricted diffusion (corresponding low signal on ADC) and not T2 shine-through effects (high signal on ADC). The reader scored the response according to 4 patterns, combining the tumor morphology on the T2-weighted images with, in case of any high signal on DWI, the specific distribution and location of the DWI signal on the restaging images. The different patterns are illustrated schematically in Figure 2 and with MRI examples in Figures 3 to 6:

a. Cases showing either a clearly normalized bowel wall at the previous tumor site without any remaining high signal on DWI (A-) or a clear bulky residual tumor mass on T2-weighted MRI with corresponding focal high signal on DWI (A+) (Figure 3);

b. Cases with primarily circular and/or irregular tumors showing irregular/spiculated fibrosis on T2-weighted MRI after CRT, either without corresponding focal high signal on DWI (B-) or with small foci of high DWI signal scattered throughout the fibrosis $(\mathrm{B}+)$ (Figure 4);

c. Cases with primarily semicircular tumors showing semicircular or focal fibrosis on T2-weighted MRI after CRT, either without any corresponding focal high signal on DWI (C-) or with focal high DWI signal, originating specifically at the inner margin of the fibrosis (C+) (Figure 5); and

d. Cases with primarily polypoid tumors showing a regression of the polyp after CRT with a focal fibrotic remnant at the site of the stalk on T2-weighted MRI, either without corresponding focal high signal on DWI (C-) or with a focal high DWI signal specifically at the site of the stalk $(\mathrm{C}+)$ (Figure 6).

If a high signal was found to be shine-through (high on ADC) or located at a different location than the specific sites described above, this was discarded as false high signal and not taken into account in the scoring to avoid false positives caused by, for example, artefacts. To study interobserver effects, a second independent reader with similar reading experience (MJL) scored a random sample of one third $(\mathrm{n}=75)$ of the study cases using the same scoring system.

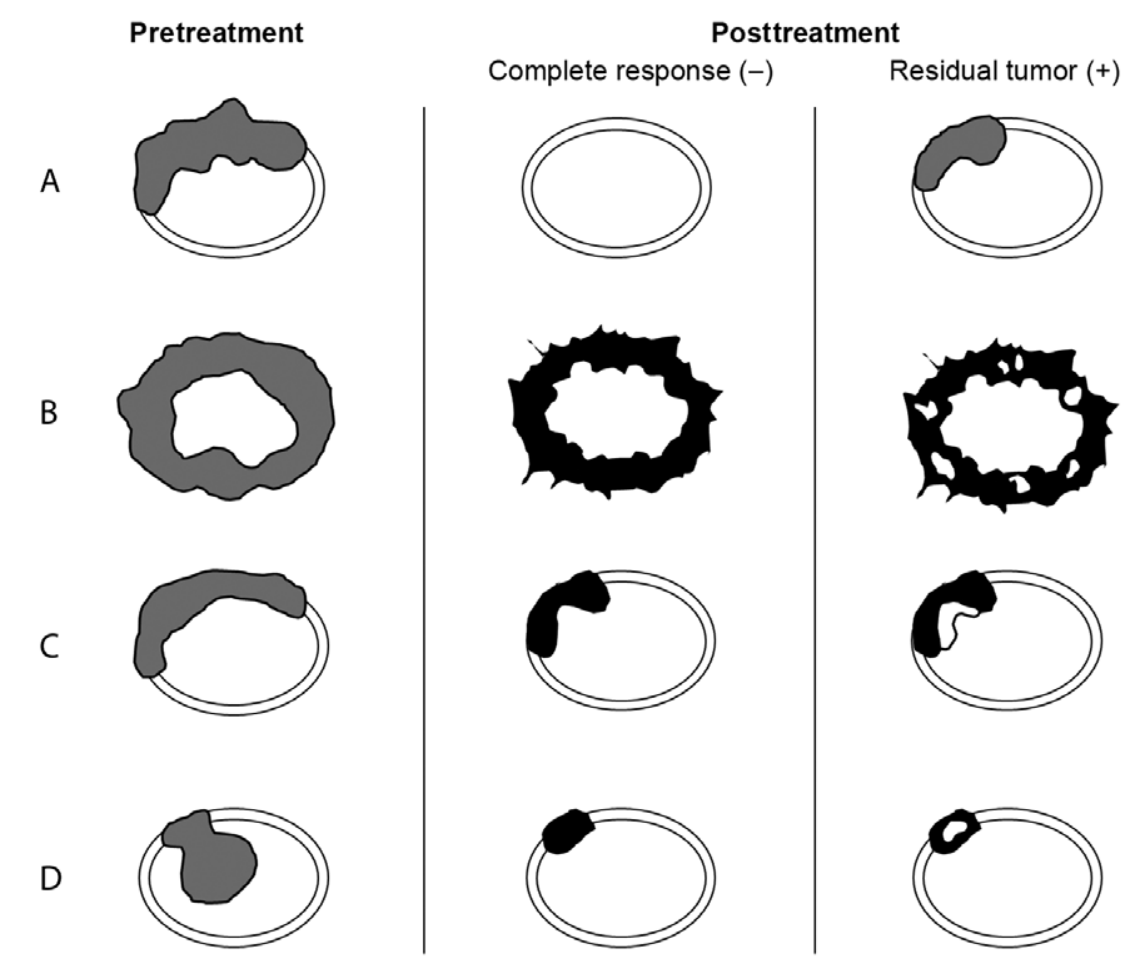

FIGURE 2. Four morphologic patterns were defined, which were each subdivided into diffusion-weighted imaging (DWI)-positive and DWInegative subpatterns. Pattern A includes cases with either a complete normalization of the rectal wall (typically observed in smaller tumors) with no high signal on DWI (A-) or a clear bulky residual tumor mass after chemoradiotherapy (CRT; A+). Pattern B includes irregular and/or circular tumors that, after CRT, show irregular and spiculated fibrosis, either without high signal on DWI (B-) or with focal spots of high signal scattered throughout the fibrosis on DWI (B+). Pattern C includes semicircular tumors that show a focal fibrotic wall thickening after CRT, either without high signal on DWI (C-) or with a focal high signal originating specifically at the inner margin of the fibrosis (C+). Pattern D includes polypoid tumors that, after CRT, show fibrotic changes at the site of the stalk of the polyp, either without high signal on DWI (D-) or with a focal high signal at the site of the stalk/fibrosis (D+). 

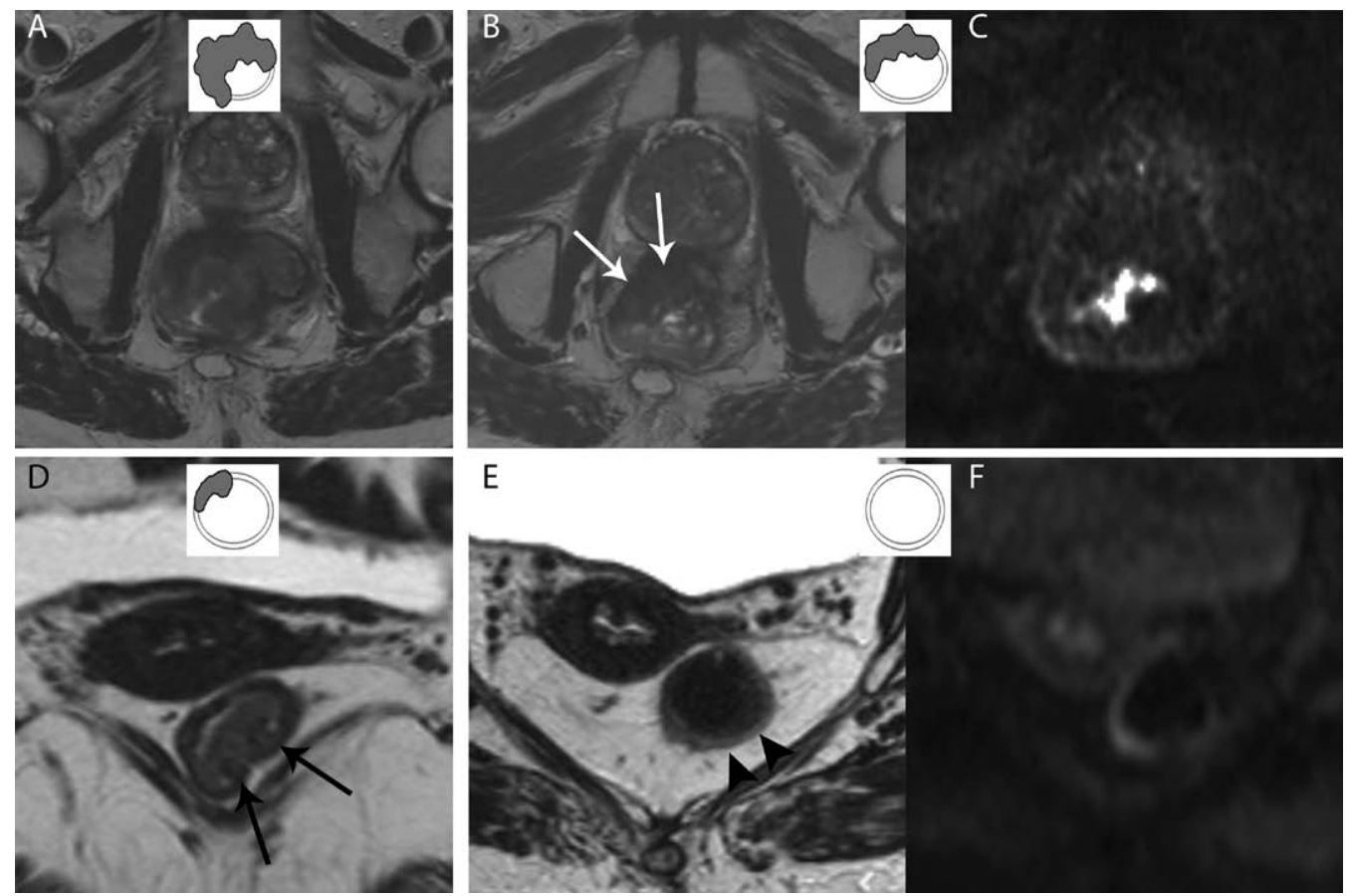

FIGURE 3. Examples of 2 pattern A cases. In the upper patient, a large bulky tumor mass is visible on pretreatment T2-weighted MRI (A). After chemoradiotherapy (CRT), the tumor has responded poorly with an obvious bulky residual tumor mass on T2-weighted MRI (arrows in B) with corresponding high signal on b1000 DWI (C). In the lower patient, a relatively small cT1 to 2 tumor is present at primary staging (arrows in D). After CRT, the rectal wall has completely normalized (arrowheads in E), and no residual mass or fibrotic changes are observed. On DWI, no focal high signal is seen $(F)$.

\section{Reference Standard}

In 161 patients, the reference standard consisted of histopathological assessment of the rectal resection specimen, including assessment of the tumor regression grade (TRG) according to Mandard et al. ${ }^{12}$ Response was dichotomized into a pathologic complete response (ypT0/ TRG1) or residual tumor (ypT1-4/TRG 2-5). The remaining 61 patients did not undergo surgery but were followed according to a watch-and-wait policy because of strong clinical evidence (on MRI, digital rectal examination, and endoscopy) of a complete tumor remission. In these patients, a recurrence-free follow-up period of $>1$ year (established by means of repeated negative MRIs and endoscopies with or without biopsies) was considered a surrogate end point of a complete response. Median recurrence-free follow-up in these patients was 36 months (range, 12-104 mo).

\section{Statistical Analyses}

Statistical analyses were performed using the Statistical Package for the Social Sciences (SPSS version 22, IBM Corps, Armonk, NY). Descriptive statistics and contingency tables were used to compare the results of the pattern-based scoring with the final response outcome (residual tumor versus complete response), and diagnostic accuracy figures were calculated. For these analyses, all DWI-positive patterns were considered patterns indicative of residual tumor, whereas all DWI-negative patterns were considered patterns indicative of a complete response. In the 75 cases that were double read by a second reader, interobserver agreement was assessed using weighted $\kappa$ values with quadratic weighting (taking into account all individual patterns ordered ascendingly according to the risk and extent of residual disease: A-, D-, C-, B-, D+, C+, $\mathrm{B}+$, and $\mathrm{A}+$ ).

\section{RESULTS}

\section{Patient Characteristics}

Baseline patient characteristics are provided in Table 2. In total, 144 patients had residual tumor after surgery $(16=y p T 1,40=y p T 2,78=y p T 3$, and $10=y p T 4)$ and 78 a complete response $(17=$ ypT0 after surgery; $61=y c T 0$ with a sustained clinical complete response during longterm watchful waiting). In the 78 complete responders, the initial tumor stage was cT1 to 2 in $19, \mathrm{cT} 3$ in 56 , and cT4 in 3. Initial nodal stage was $\mathrm{cN} 0$ in $27, \mathrm{cN} 1$ in 26 , and $\mathrm{cN} 2$ in 25 patients; 2 (3\%) of the 78 complete tumor responders still had node-positive disease and underwent total mesorectal excision (which confirmed a pN1 stage in both patients). The median interval between restaging MRI and surgery in the operated patients was 18 days (range, 1-50 d). 

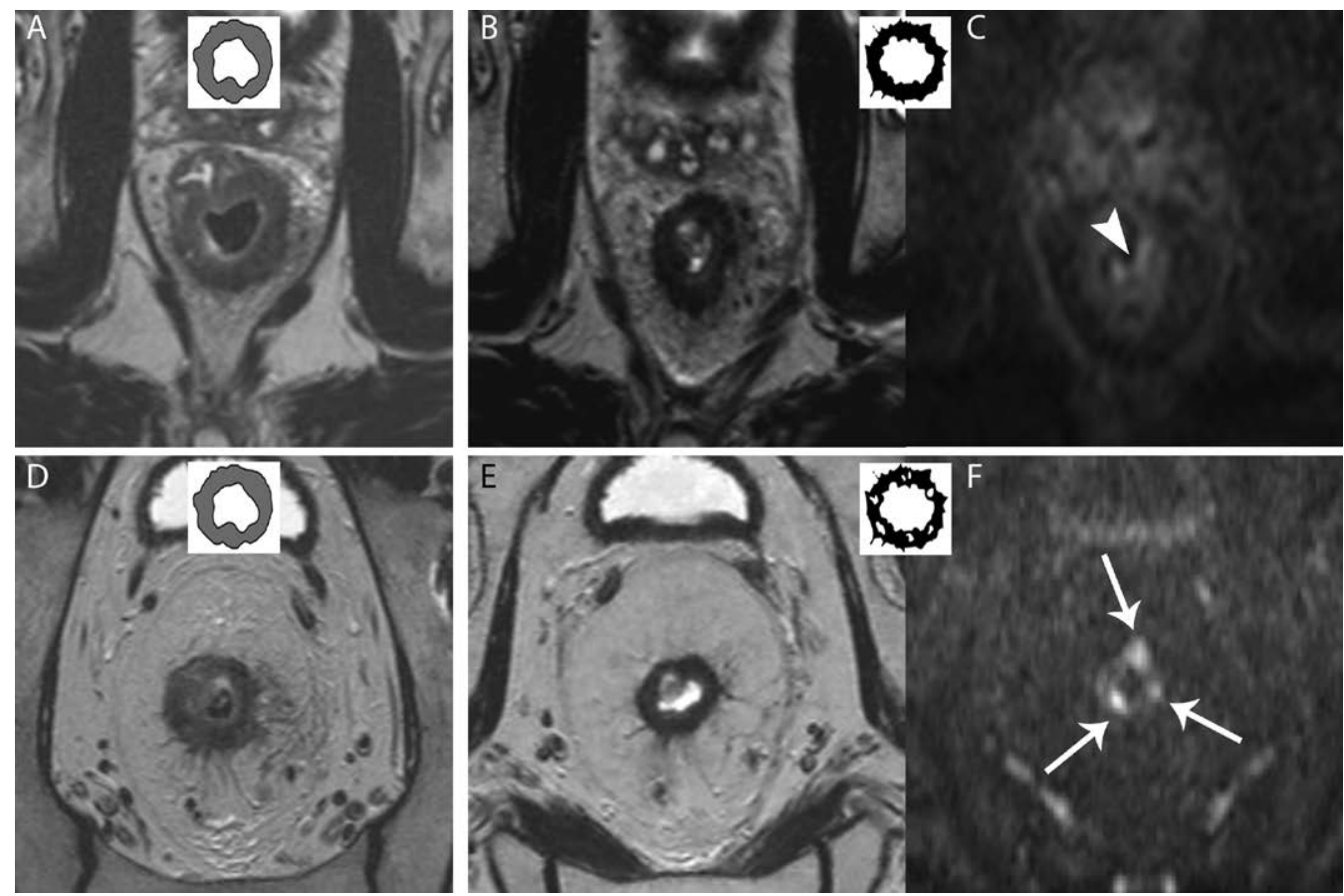

FIGURE 4. Examples of 2 pattern $B$ cases. On pretreatment T2-weighted MRI ( $A$ and $D)$, both patients have irregular circular tumors, in the lower case (D) with spiculation. On the T2-weighted images after chemoradiotherapy (CRT; B and E), both tumors show irregular fibrotic

changes with spiculation. In the upper patient, no signal is visualized in the rectal wall at the site of the fibrosis (B-) and only a slight area of T2 shine-through is visualized caused by fluid in the lumen (arrowhead in C). In the lower patient, several spots of high signal are visible scattered throughout the fibrosis (B+; arrows in F).

\section{Diagnostic Performance}

Table 3 shows the diagnostic accuracy figures for the different response patterns. Sensitivity for the pattern-based approach to discriminate tumor from complete response was $94 \%$, specificity $77 \%$, positive predictive value $88 \%$, negative predictive value (NPV) $87 \%$, and overall accu-
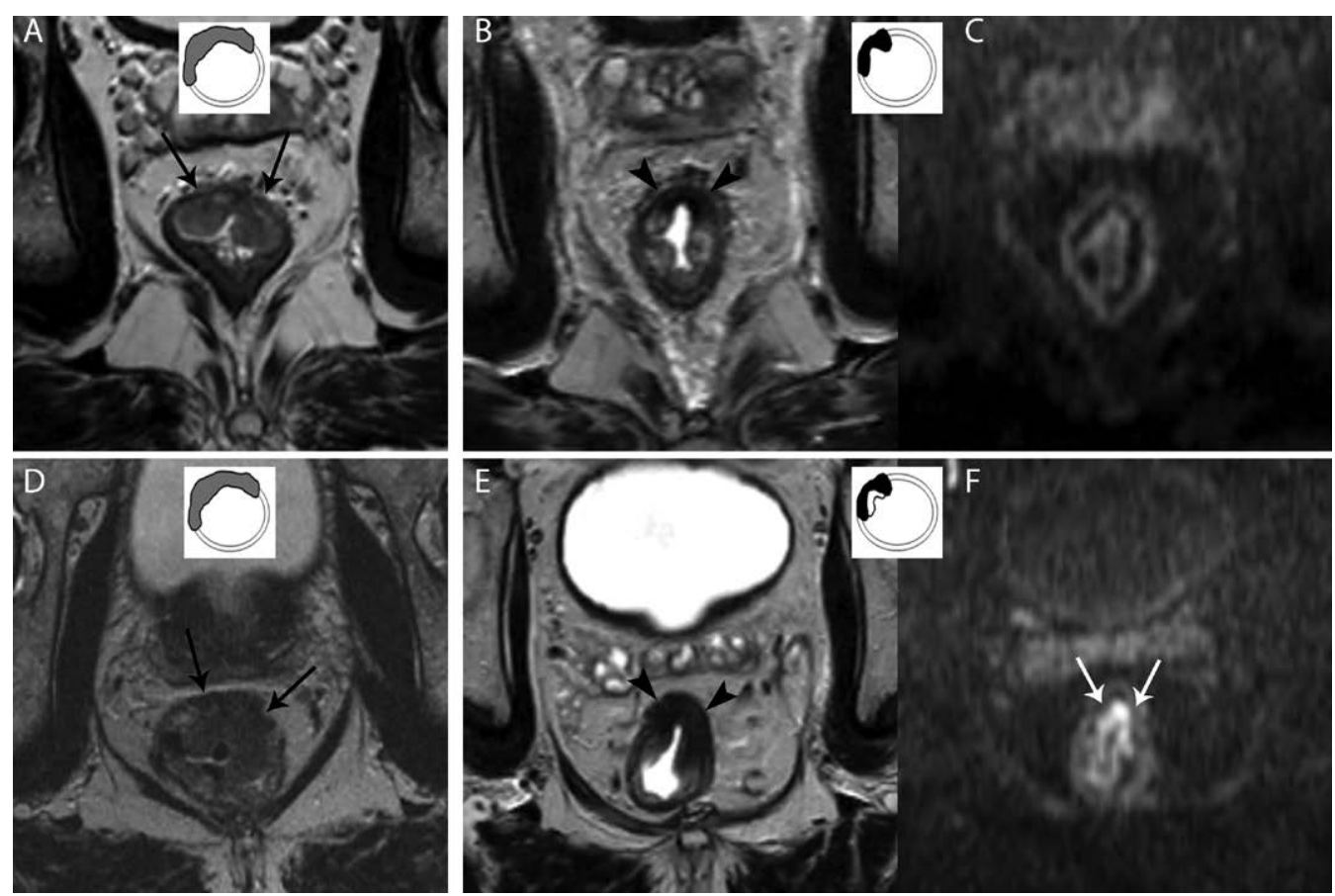

FIGURE 5. Examples of 2 pattern $C$ cases. On pretreatment T2-weighted MRI, both patients have a semicircular tumor originating from the anterior rectal wall (arrows in A and D). On the T2-weighted images after chemoradiotherapy (CRT) both tumors show a similar fibrotic wall thickening (arrowheads in B and E). In the upper patient, no signal is visualized in the rectal wall at the site of the fibrosis (C-). In the lower patient, a clear high signal is visualized at the inner margin of the fibrosis ( $\mathrm{C}+$; arrows in $\mathrm{F}$ ). 

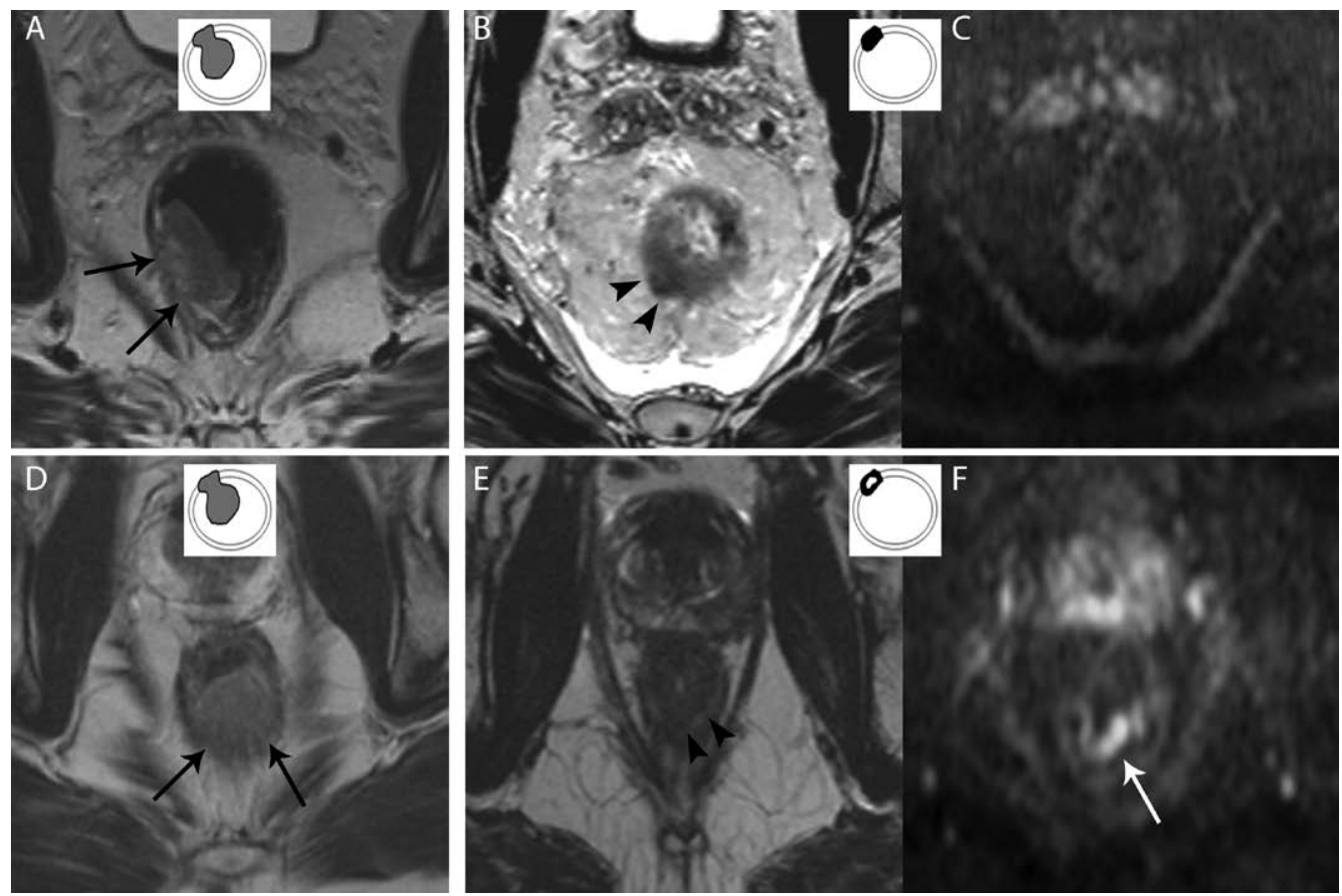

FIGURE 6. Examples of 2 pattern D cases. On pretreatment T2-weighted MRI, both patients have a polypoid tumor (arrows in A and D). On the T2-weighted images after chemoradiotherapy (CRT), both tumors show some slight fibrotic changes in the rectal wall (arrowheads in B and E) at the site of the stalk of the polyp. In the upper patient (C), no signal is visualized at the site of the fibrosis on DWI (D-). In the lower patient, a clear high signal is visualized at the site of the fibrosis, at the site of the stalk of the polyp (D+; arrow in F).

racy $88 \%$. Pattern A was $100 \%$ sensitive to detect residual tumor $(\mathrm{A}+)$ and $100 \%$ specific to identify a complete response (A-). Among the remaining patterns, results were better for pattern C (semicircular fibrosis: accuracy 86\%) and pattern D (polypoid: accuracy 92\%) compared with pattern B (irregular fibrosis: accuracy 74\%).

\section{Distribution of Patterns per Tumor Stage}

Table 4 shows the distribution of patterns according to the initial (pretreatment) $\mathrm{cT}$ stage. In patients with initial stage cT4 tumors, no DWI-negative patterns occurred, and the majority (59\%) showed obvious bulky residual tumors (pattern A+) after CRT. Only 3 initial cT4 patients underwent a complete response, but all 3 had irregular fibrosis with suspected residual tumor on DWI (pattern $\mathrm{B}+$ ). The majority of cT1 to 2 tumors had semicircular (pattern C) tumors, of which most underwent a complete response (pattern $\mathrm{C}-$ ). A completely normalized rectal wall (pattern A-) occurred in $10 \%$ of cT1 to 2 patients, $6 \%$ of $\mathrm{CT} 3$ patients, and in none of the cT4 patients.

\section{False-Positive and False-Negative Cases}

Eighteen false-positive and 9 false-negative cases occurred. Apart from 1 false-positive and 1 false-negative case in patients with initial stage cT1 to 2 tumors, these exclusively occurred in patients with initial stage cT3 to 4 tumors. Of the 9 false-negative cases, 5 were near-complete responders (TRG 2). In 2 patients, the rectal wall was collapsed at the previous tumor site on restaging MRI, making the response pattern more difficult to assess. In the remaining cases, the main reasons for error were small susceptibility artefacts caused by air in the rectal lumen occurring at the site of the tumor.

\section{Interobserver Agreement}

Interobserver agreement was good, with a $\kappa$ of $0.75(95 \%$ CI, 0.63-0.87).

\section{DISCUSSION}

The aim was to evaluate the performance of a patternbased approach to differentiate between a complete tumor response and residual tumor after neoadjuvant treatment for rectal cancer combining the morphology on T2-weighted MRI with distinct signal patterns on diffusion-weighted MRI. In patients with a clear bulky residual tumor mass or a complete normalization of the rectal wall, sensitivity and specificity were $100 \%$. These clear-cut patterns will typically not offer much challenge in daily practice but only occurred in a minority of patients, with $21 \% \mathrm{~A}+$ (mainly in the case of initial cT4 stage) and $6 \%$ A-. The remaining patients constitute the more clinically challenging cases, in which a pattern-based approach may be of added value. In these patients, the use of specific morphology-DWI patterns resulted in good diagnostic performance, with overall accuracies ranging between 
TABLE 2. Baseline patient and treatment characteristics

\begin{tabular}{|c|c|}
\hline Variable & No. of Patients (total $N=222$ ) \\
\hline \multicolumn{2}{|l|}{ Sex } \\
\hline Men & 154 \\
\hline Women & 68 \\
\hline Median age (range), y & $66(31-88)$ \\
\hline \multicolumn{2}{|l|}{$\begin{array}{l}\text { Primary cTN stage (as assessed with } \\
\qquad M \mathrm{MR}^{\mathrm{a}} \text { ) }\end{array}$} \\
\hline \multicolumn{2}{|l|}{ CT stage } \\
\hline cT1 to 2 & 29 \\
\hline cT3 & 171 \\
\hline cT4 & 22 (1 cT4a, 21 cT4b) \\
\hline \multicolumn{2}{|l|}{ cN stage } \\
\hline $\mathrm{cNO}$ & 47 \\
\hline $\mathrm{cN} 1$ & 63 \\
\hline cN2 & 112 \\
\hline \multicolumn{2}{|l|}{$\begin{array}{l}\text { Restaging ycTN stage after CRT (as } \\
\text { assessed with MRIa) }\end{array}$} \\
\hline \multicolumn{2}{|l|}{ ycT stage } \\
\hline уст0 & 70 \\
\hline ycT1 to 2 & 39 \\
\hline уст3 & 92 \\
\hline уст4 & 21 (3 cТ4a, 18 cT4b) \\
\hline \multicolumn{2}{|l|}{ ycN stage } \\
\hline ycNo & 143 \\
\hline ycN1 & 54 \\
\hline ycN2 & 25 \\
\hline \multicolumn{2}{|l|}{ Final surgical treatment } \\
\hline Total mesorectal excision & 145 \\
\hline Transanal endoscopic microsurgery & 16 \\
\hline None (watch and wait) & 61 \\
\hline \multicolumn{2}{|l|}{ Final ypTN/ycTN stage } \\
\hline \multicolumn{2}{|l|}{ урT stage/ycT stage } \\
\hline урT0/усто & 78 \\
\hline урT1 & 16 \\
\hline урт2 & 40 \\
\hline уртз & 78 \\
\hline урТ4 & 10 \\
\hline \multicolumn{2}{|l|}{ ypN stage/ycN stage } \\
\hline ypNO/ycNO & 165 \\
\hline yN1 & 46 \\
\hline yN2 & 11 \\
\hline \multicolumn{2}{|l|}{ Final tumor response $\mathrm{e}^{\mathrm{b}}$} \\
\hline Complete response & 78 \\
\hline Residual tumor & 144 \\
\hline
\end{tabular}

aThe MRI stages were derived from the original clinical MRI reports in the hospital's patient database system.

bIn 161 patients, response and final yTN stage was assessed based on histopathology of the resection specimen after surgery. In the remaining 61 patients (all from the complete response group), the ycTO and ycNO stage was based on a sustained clinical complete response (with repeated negative findings without signs of recurrence on $\mathrm{MRI}$, endoscopy, and digital rectal examination) with a median recurrence-free follow-up period of 36 months (minimum of $1 \mathrm{y}$ ).

$74 \%$ and $92 \%$. For the whole patient group, overall accuracy was $88 \%$, which is similar to the accuracies ranging between $82 \%$ and $90 \%$ reported previously by different groups for visual evaluation of DWI without a specific pattern-based approach. ${ }^{2,3,13}$ Sensitivity (94\%) and positive predictive value (88\%) in our study are also within the same range as reported previously (81-98\%)., 2,3,13 However, specificity and NPV in our study were considerably better. Our overall NPV was $87 \%$ compared with $43 \%$ to $81 \%$ reported previously. ${ }^{2,3,13}$ Specificity in our study was $77 \%$ compared with $52 \%$ to $64 \%$ reported previously by Lambregts et $\mathrm{al}^{3}$ and $33 \%$ to $50 \%$ by Song et al. ${ }^{13}$ Only the group of Kim et $\mathrm{al}^{2}$ reported a higher specificity of $82 \%$ to $91 \%$. Compared with a visual DWI evaluation without any specific criteria or patterns, our approach may thus particularly be of value to rule out tumor and to help identify patients with a complete tumor response. Mainly patterns C (semicircular or focal fibrosis after CRT) and D (polypoid tumors with fibrosis at the site of the stalk after CRT) proved beneficial, because these showed the best concordance with the final response outcome. The key principle of patterns $\mathrm{C}$ and $\mathrm{D}$ is that, in case of a high signal on DWI, this occurs at a specific site (originating at the inner margin of the fibrosis in pattern $\mathrm{C}$ and in the stalk of the polyp in pattern $\mathrm{D}$ ). Knowing where to specifically look for a high DWI signal apparently helps to more accurately recognize areas of residual tumor and to discriminate these from high DWI signal caused, for example, by artefacts that might otherwise be falsely interpreted as suspicious for a residual tumor. With this approach, the risk of missing a residual tumor, which would result in undertreatment and thus a risk for recurrence when selecting these patients for watch and wait, is relatively low (5\% in pattern $\mathrm{C}$ and $8 \%$ in pattern $\mathrm{D}$ ), albeit not nonnegligible.

Conversely, in pattern B (circular tumors with irregular/spiculated fibrosis), results were considerably poorer. Only 3 DWI-negative pattern B cases were observed, of which none corresponded with an actual complete response. On the other hand, 9 false positives occurred. Apparently when we see irregular/spiculated fibrosis after CRT, the signal pattern on DWI is less reliable. This is probably because in these cases any remaining areas of vital tumor are more scattered (as small tumor nests) throughout the fibrosis, making them more difficult to assess on DWI. In the majority (80\%) of the pattern B cases, residual tumor was still present, indicating that it may in fact even be the safest option to consider all these patients to be at risk for residual tumor, albeit at the expense of overestimating tumor in $20 \%$. The main problem of overestimating tumor is that patients will be denied the option of watch and wait, despite being potential suitable candidates. In the current study, 17 patients with a complete response underwent surgical resection. This is in part because, in the early study period, watch and wait was not yet routinely offered as a treatment option. Moreover, even with an optimal clinical response assessment using a combination of MRI (including DWI), digital rectal examination, and endoscopy, $\approx 15 \%$ of complete responders will typically still be missed. ${ }^{14}$

With the current study we only focused on response assessment of the primary tumor and did not take the nodal stage into account. However, we fully acknowledge 
TABLE 3. Distribution of response patterns and corresponding diagnostic performance to differentiate between a complete response and residual tumor

\begin{tabular}{|c|c|c|c|c|c|c|c|}
\hline \multirow[b]{2}{*}{ Pattern } & \multicolumn{2}{|c|}{ Final response } & \multicolumn{5}{|c|}{ Diagnostic performance, $\%$} \\
\hline & Residual tumor $(N=144)$ & Complete response $(N=78)$ & Sensitivity & Specificity & $P P V$ & $N P V$ & Accuracy \\
\hline \multicolumn{8}{|c|}{ Pattern A } \\
\hline+ & $46(\mathrm{TP})$ & $0(\mathrm{FP})$ & 100 & 100 & 100 & 100 & 100 \\
\hline- & $0(\mathrm{FN})$ & $14(\mathrm{TN})$ & & & & & \\
\hline \multicolumn{8}{|c|}{ Pattern B } \\
\hline+ & $34(\mathrm{TP})$ & 9 (FP) & 92 & 0 & 79 & 0 & 74 \\
\hline- & $3(\mathrm{FN})$ & $0(\mathrm{TN})$ & & & & & \\
\hline \multicolumn{8}{|l|}{ Pattern C } \\
\hline+ & $51(\mathrm{TP})$ & $9(\mathrm{FP})$ & 91 & 81 & 85 & 88 & 86 \\
\hline- & $5(\mathrm{FN})$ & $38(\mathrm{TN})$ & & & & & \\
\hline \multicolumn{8}{|c|}{ Pattern D } \\
\hline+ & $4(\mathrm{TP})$ & 0 (FP) & 80 & 100 & 100 & 89 & 92 \\
\hline- & $1(\mathrm{FN})$ & $8(\mathrm{TN})$ & & & & & \\
\hline Total & $135 \mathrm{TP}$ & $18 \mathrm{FP}$ & 94 & 77 & 88 & 87 & 88 \\
\hline
\end{tabular}

Note that all TP and FN cases were histopathologically proven after surgical resection. Of the 60 TN cases, 8 were ypT0 after surgery and 52 were clinical complete responders followed according to a watch-and-wait policy. Of the 18 FP cases, 9 were ypT0 after surgery and 9 were clinical complete responders followed according to a watch-and-wait policy.

$\mathrm{TN}=$ true negative; $\mathrm{FN}=$ false negative; $\mathrm{FP}=$ false positive; $\mathrm{TP}=$ true positive; $\mathrm{PV}=$ positive predictive value; $\mathrm{NPV}=$ negative predictive value

that a complete response assessment should also include an evaluation of the nodes. When considering patients for watch and wait, we need to ensure that, apart from the primary tumor, all of the suspicious lymph nodes have resolved. In the current cohort, 2 (3\%) of the 78 complete tumor responders still had node-positive disease, which was recognized on restaging MRI. Both patients were operated on, confirming a pN1 status.

There are some limitations to our study design. First, this was a retrospective study, and the patterns described in this study will need to be tested prospectively. Moreover, although we have shown that, in the hands of experienced readers, results for the pattern approach are reproducible with a $\kappa$ of 0.75 , reproducibility of the findings will also need to be established for less-expert readers. Second, the percentage of complete responders in our study is relative- ly high (35\%). This is because patients were included from a referral center for organ preservation to which patients with a suspected good response are referred for additional response evaluation. Moreover, the availability of organ preservation as a treatment option at the institution may have induced a certain preference for CRT, particularly in elderly or frail patients. Third, our study did not include a direct comparison of routine visual interpretation of DWI and the pattern-based approach. As such, the added benefit of the pattern approach was estimated by comparing our results with those reported previously in literature. Fourth, 10 patients had to be excluded because of severe artefacts on DWI (mainly caused by metal hip replacements), rendering the diffusion-weighted images uninterpretable. This is a known drawback of DWI that may limit its clinical use in certain patients. ${ }^{7}$ Finally, there were some

Table 4. Distribution of patterns per tumor stage

\begin{tabular}{|c|c|c|c|c|c|c|c|c|}
\hline \multirow{4}{*}{$\begin{array}{l}\text { Initial tumor stage } \\
\text { (before treatment) } \\
\text { cT1-2 }(\mathrm{N}=29)\end{array}$} & \multicolumn{8}{|c|}{ Pattern of response vs final outcome } \\
\hline & & & \multicolumn{2}{|c|}{ Final outcome } & & & \multicolumn{2}{|c|}{ Final outcome } \\
\hline & \multicolumn{2}{|c|}{ DWI-patterns } & $C R(T N)$ & Tumor (FN) & \multicolumn{2}{|c|}{ DWI+ patterns } & $C R(F P)$ & Tumor (TP) \\
\hline & A- & $3(10 \%)$ & 3 & - & $A+$ & $1(3 \%)$ & - & 1 \\
\hline & B- & $0(0 \%)$ & - & - & $\mathrm{B}+$ & $0(0 \%)$ & - & - \\
\hline & $\mathrm{C}_{-}$ & $14(48 \%)$ & 13 & 1 & $\mathrm{C}+$ & $7(24 \%)$ & 1 & 6 \\
\hline & D- & $2(7 \%)$ & 2 & - & $\mathrm{D}+$ & $2(7 \%)$ & - & 2 \\
\hline \multirow[t]{4}{*}{ CT3 $(\mathrm{N}=171)$} & A- & $11(6 \%)$ & 11 & - & $A+$ & 32 (19\%) & - & 32 \\
\hline & B- & $3(2 \%)$ & - & 3 & $\mathrm{~B}+$ & $36(21 \%)$ & 6 & 30 \\
\hline & $\mathrm{C}_{-}$ & $29(17 \%)$ & 25 & 4 & $\mathrm{C}+$ & $51(30 \%)$ & 8 & 43 \\
\hline & D- & 7 (4\%) & 6 & 1 & $\mathrm{D}+$ & $2(1 \%)$ & - & 2 \\
\hline \multirow[t]{4}{*}{ cT4 $(\mathrm{N}=22)$} & A- & $0(0 \%)$ & - & - & $\mathrm{A}+$ & 13 (59\%) & - & 13 \\
\hline & B- & $0(0 \%)$ & - & - & $\mathrm{B}+$ & $7(32 \%)$ & 3 & 4 \\
\hline & C- & $0(0 \%)$ & - & - & $\mathrm{C}_{+}$ & $2(9 \%)$ & - & 2 \\
\hline & D- & $0(0 \%)$ & - & - & $\mathrm{D}+$ & $0(0 \%)$ & - & - \\
\hline
\end{tabular}

Numbers are absolute numbers; percentages are given in parentheses. Note that the initial tumor stages were determined on the initial staging MRI. $\mathrm{CR}=$ complete response; $\mathrm{TN}=$ true negative; $\mathrm{FN}=$ false negative; $\mathrm{FP}=$ false positive; $\mathrm{TP}=$ true positive. 
variations in the DWI acquisition and patient preparation protocols used throughout the study (as a result of protocol optimization over the years). This may have affected our study results, although we believe that this effect will probably be limited given the study setting where only images with the same b-value (b1000) were assessed and no quantitative measurements were performed. The use of nonstandardized imaging protocols may even be viewed as a positive factor, because it suggests that results may be generalized and will likely be less dependent on protocol variations between centers/scanners.

\section{CONCLUSION}

A pattern-based approach, taking into account the morphology on T2-weighted MRI combined with distinct DWI signal patterns, can be helpful to assess response after neoadjuvant treatment in patients with rectal cancer and aid in identifying patients with a complete tumor response after CRT. These patterns may serve as a basis to help teach radiologists to read DWI after CRT and to help improve their diagnostic performance in assessing response of rectal tumors on MRI.

\section{REFERENCES}

1. van der Paardt MP, Zagers MB, Beets-Tan RG, Stoker J, Bipat S. Patients who undergo preoperative chemoradiotherapy for locally advanced rectal cancer restaged by using diagnostic MR imaging: a systematic review and meta-analysis. Radiology. 2013;269:101-112.

2. Kim SH, Lee JM, Hong SH, et al. Locally advanced rectal cancer: added value of diffusion-weighted MR imaging in the evaluation of tumor response to neoadjuvant chemo- and radiation therapy. Radiology. 2009;253:116-125.

3. Lambregts DM, Vandecaveye V, Barbaro B, et al. Diffusionweighted MRI for selection of complete responders after chemoradiation for locally advanced rectal cancer: a multicenter study. Ann Surg Oncol. 2011;18:2224-2231.

4. Martens MH, Maas M, Heijnen LA, et al. Long-term outcome of an organ-preservation program after neoadjuvant treatment for rectal cancer. J Natl Cancer Inst. 2016;108:pii:djw171
5. Habr-Gama A, Gama-Rodrigues J, São Julião GP, et al. Local recurrence after complete clinical response and watch and wait in rectal cancer after neoadjuvant chemoradiation: impact of salvage therapy on local disease control. Int J Radiat Oncol Biol Phys. 2014;88:822-828.

6. Koh DM, Blackledge M, Padhani AR, et al. Whole-body diffusion-weighted MRI: tips, tricks, and pitfalls. AJR Am J Roentgenol. 2012;199:252-262.

7. Sánchez- González J. How to identify and avoid artefacts on DWI In: Luna A, Ribes R, Soto JA, eds. Diffusion MRI Outside the Brain: A Case-Based Review and Clinical Applications. Berlin, Heidelberg, Germany: Springer-Verlag; 2012:17-31.

8. Taylor FG, Swift RI, Blomqvist L, Brown G. A systematic approach to the interpretation of preoperative staging MRI for rectal cancer. AJR Am J Roentgenol. 2008;191:1827-1835.

9. Lambregts DM, Maas M, Bakers FC, et al. Long-term followup features on rectal MRI during a wait-and-see approach after a clinical complete response in patients with rectal cancer treated with chemoradiotherapy. Dis Colon Rectum. 2011;54:1521-1528.

10. Kim SH, Yoon JH, Lee Y. Added value of morphologic characteristics on diffusion-weighted images for characterizing lymph nodes in primary rectal cancer. Clin Imaging. 2015;39:1046-1051.

11. Nasu K, Kuroki Y, Minami M. Diffusion-weighted imaging findings of mucinous carcinoma arising in the ano-rectal region: comparison of apparent diffusion coefficient with that of tubular adenocarcinoma. Jpn J Radiol. 2012;30:120-127.

12. Mandard AM, Dalibard F, Mandard JC, et al. Pathologic assessment of tumor regression after preoperative chemoradiotherapy of esophageal carcinoma. Clinicopathologic correlations. Cancer. 1994;73:2680-2686.

13. Song I, Kim SH, Lee SJ, Choi JY, Kim MJ, Rhim H. Value of diffusion-weighted imaging in the detection of viable tumour after neoadjuvant chemoradiation therapy in patients with locally advanced rectal cancer: comparison with T2 weighted and PET/CT imaging. Br J Radiol. 2012;85:577-586.

14. Maas M, Lambregts DM, Nelemans PJ, et al. Assessment of clinical complete response after chemoradiation for rectal cancer with digital rectal examination, endoscopy, and MRI: selection for organ-saving treatment. Ann Surg Oncol. 2015;22:3873-3880. 